\section{An improved measurement technique for} human operator stability in aiming tasks

\section{HADANI and Y. BERGMAN \\ Technion, Israel Institute of Technology, Haifa, Israel}

A technique is presented for field measurement of human operator stability in an aiming task. The small angular displacements of the aiming axis are measured by photographing a part of the target as reflected by a mirror mounted on the aiming device.

Steadiness is required when one must maintain a fixed posture, aim at a target, or accurately trace a pattern. Alain and Avon (1976) indicate the inadequacy of existing laboratory instruments, stabilometers and steadiness testers, in real-life situations. They present a measurement technique that can be easily applied to experimental conditions simulating real-life conditions. In their technique, a still photograph of an infrared diode attached to the end of a rifle barrel is taken. The stability score is defined as the total area covered by the diode's image on the film during aiming. While this technique yields an overall stability score, it does not provide exact information on the spatial orientations of the aiming axis or the momentary deviations from the target of that axis.

In this paper, we present an improved measurement technique for the evaluation of the human operator stability that overcomes these difficulties. A succession of movie photographs of part of the target as reflected by a mirror mounted on the aiming device is taken. Thus the small angular displacements of the aiming axis between two successive frames can be measured.

\section{APPARATUS}

A high-quality 7-cm-diam optically flat, front-surface mirror is mounted under the barrel near the center of gravity of an air rifle. The rifle is a spring-operated type, mounted on a fixed-head tripod. Spring-loaded screws allow independent vertical and horizontal alignment of the mirror mount. The $150 \mathrm{~g}$ added weight of the mirror and mount is a small percentage of the uninstrumented weight.

A square target $(1.5 \times 1.5 \mathrm{~m})$ with a 14-cm-diam hole in its center is divided into a matrix of uniquely defined rectangles, each $3 \mathrm{~cm}$ high $\times 6 \mathrm{~cm}$ long. The target is positioned $30 \mathrm{~m}$ in front of the mirror mount.

The authors are indebted to Adam Ringer for his helpful suggestions and to the photographer, Israel Haramati, for solving many of the technical problems encountered in the realization of the apparatus. I. Hadani is with the Department of Biomedical Engineering. Y. Bergman is with the Faculty of Industrial and Management Engineering.
Its center height is approximately that of the mirror center. A 16-mm Bolex Model H-16 camera is attached to a $1,200-\mathrm{mm}-\mathrm{fl}$ 3.5-in. Questar telescope that allows one to view the mirror mount through the target center hole. The film is Kodak Negative Plus-X Number 7231. The telescope is focused on the target rectangles $60 \mathrm{~m}$ away. Each rectangle subtends $.5 \times 1$ milliradians within the camera's field of view. Thirty meters behind the barrel mount mirror, there is a dual-incandescent lamp projector activated by the cessation of the aiming task by means of a trigger-mounted microswitch.

\section{PROCEDURE AND DATA REDUCTION}

For each subject, the tripod height is adjusted to suit the subject's height and the rifle sight is centered on target. Thereafter, the mirror on the barrel is aligned per the requirement that the telescope "sees itself" centered in its field of view. Following the tripod trial, the tripod is removed from the rifle and the subject is instructed to maintain the rifle's approximate position in space. The subject then repeats the aiming task with

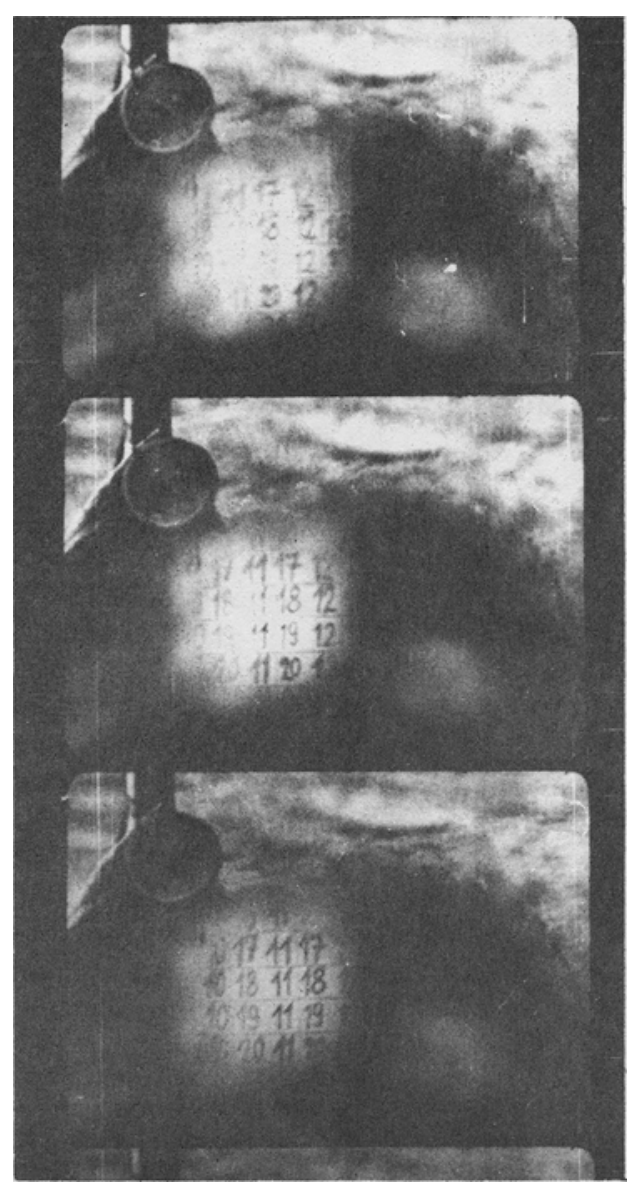

Figure 1. Representative filmed data obtained by using the measurement technique described in the text. 


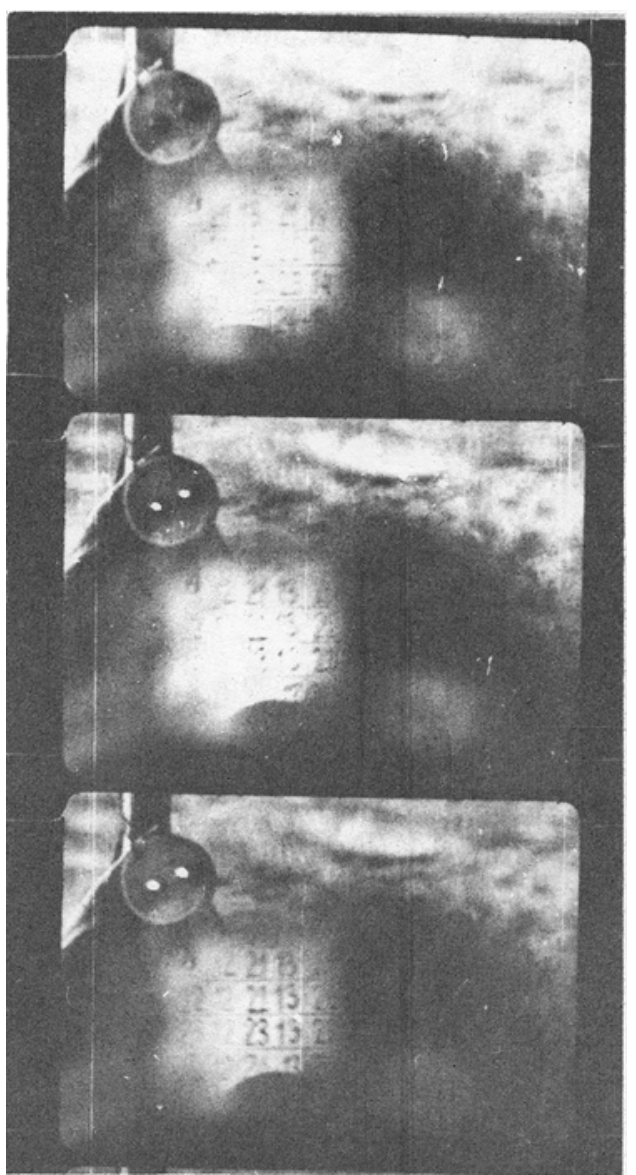

Figure 2. Same as Figure 1; the activation of the incandescent lamp (middle frame) and the reflected telescope can be seen.

the free rifle. The camera is operated at 24 frames/sec, beginning shortly before the trial and ending when the incandescent lamp indicates completion. Following each aiming task completion (trigger squeeze), the mirror alignment is rechecked.

Figures 1 and 2 show representative filmed data frames. The data itself are examined from the projected images. Analysis involves the 24 frames prior to trigger pull. The precision of measurement is to the nearest .25 milliradian. Results shown in Figures 3 and 4 are for each of two subjects. Note that the intersection of the axis is not the center of target but the mean value of the horizontal and vertical data points. The true target center can be determined only if the telescope itself appears in a frame.

\section{DISCUSSION}

The measurement technique presented here provides information about the spatial orientation of the aiming axis as well as the momentary deviation from target center of that axis. In its present form, this technique is insensitive to horizontal and vertical translatory movements in the frontal plane. Thus an exact determination of the momentary spatial orientation of the aiming axis is not possible. The difficulty can be overcome by simultaneous measurement of these translatory movements. This can be done by using an additional camera-mounted

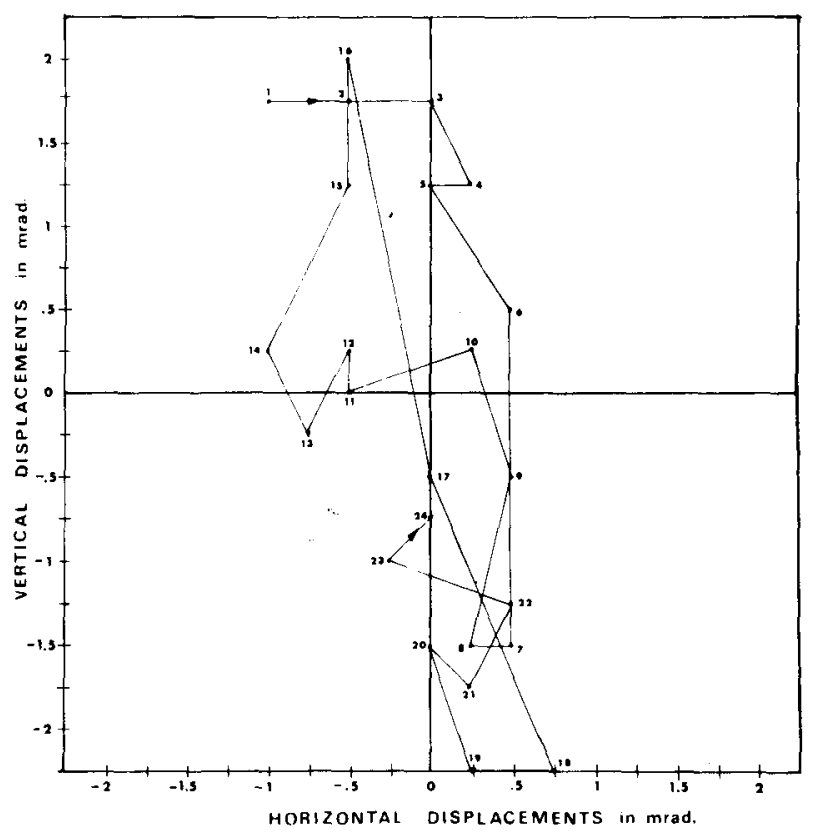

Figure 3. Results obtained for the last second (24 frames) before trigger squeeze.

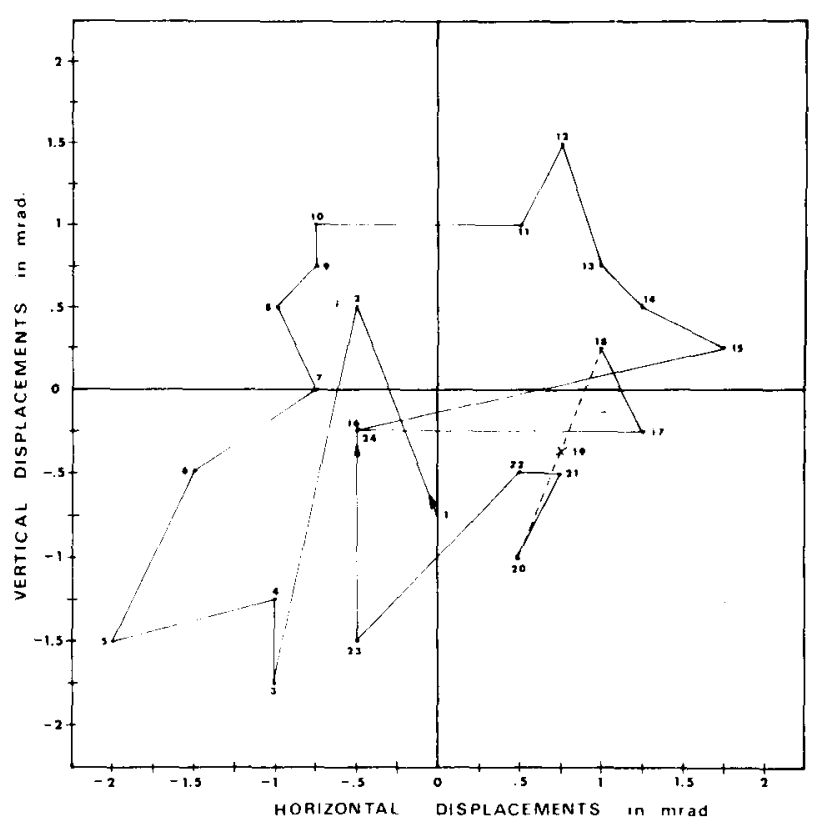

Figure 4. Same as Figure 3 for a different subject. Data Point 19 could not be read because of a blurred frame. 
telescope that is focused at some marked spot on the aiming device, preferably on the mirror itself. As for rotations around the aiming axis and translatory movements along that axis, their contribution to measurement error is of the second order and therefore can be neglected.

The main advantages of our measurement technique are its relative simplicity and its applicability to field situations. An additional advantage is its high accuracy compared with existing techniques. As a matter of fact, the measurement resolution is limited by the linespread function of the optics and modified by the combined degradation of the film and subsequent processes. Without undue difficulty, a resolution of .1 milliradian can be obtained. This degree of accuracy makes our technique suitable for research on the influences of various parameters such as posture, weight distribution, and sight structure on performance.

\section{REFERENCES}

Alain, C., \& Avon, G. A measurement technique for stability as applied in rifle shooting. Behavior Research Methods \& Instrumentation, 1976, 8, 5-7.

(Received for publication July 26, 1979; revision accepted April 2, 1980.) 